\title{
Tecnologia para apoio a assistência de enfermagem às mulheres em situação de violência sexual
}

Technology to support nursing care for women in situations of sexual violence Tecnología para apoyo a la atención de enfermería a mujeres en situación de violencia sexual

Odelle Mourão Alves ${ }^{1}$ ๑ nttps://orcid.org/0000-0002-8590--3780

Cândida Caniçali Primo ${ }^{1}$ ic nttps://orcid.org/0000-0001-5141-2898

Fábio Lúcio Tavares ${ }^{1}$ ic https://orcid.orgy/0000-0002-4725-0897

Eliane de Fátima Almeida Lima' io nttps://orcid.org/0000-0001-5128-3715

Franciéle Marabotti Costa Leite' ${ }^{1}$ io nttps://orcid.org/0000-0002-6171-6972

Como citar:

Alves OM, Primo CC, Tavares FL, Lima EF, Leite FM. Tecnologia para apoio a assistência de enfermagem às mulheres em situação de violência sexual. Acta Paul Enferm. 2021;34:eAPE001085.

DOI

http://dx.doi.org/10.37689/actaape/2021A0001085

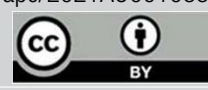

Descritores

Tecnologia em saúde; Aplicativos móveis; Processo de enfermagem; Cuidados de enfermagem;

Violência sexual

Keywords

Biomedical technology; Mobile applications; Nursing process; Nursing care; Sex offenses

Decriptores

Tecnología biomédica; Aplicaciones móviles; Atención de enfermeira; Delitos sexuales; Proceso

de enfermería

Submetido

9 de Maio de 2020

Aceito

1 de Março de 2020

\section{Autor correspondente}

Odelle Mourão

E-mail: odellealves@hotmail.com

\section{Resumo}

Objetivo: Desenvolver um aplicativo para apoio ao processo de enfermagem na assistência à mulher em situação de violência sexual.

Métodos: Pesquisa aplicada de produção tecnológica dividida em três etapas: elaboração do material teórico por meio de revisão de literatura; avaliação dos diagnósticos e intervenções de enfermagem; e desenvolvimento do aplicativo móvel.

Resultados: 0 aplicativo "CuidarTech ${ }^{\circledR}$ EnfPorElas" apresenta três opções de navegação: Diagnósticos e intervenções de enfermagem"- com 33 diagnósticos e 613 intervenções de enfermagem, correlacionados e relevantes para assistência à mulher em situação de violência sexual; "Processo de Enfermagem" - exibe conteúdos teóricos sobre o Processo de Enfermagem; e "Créditos" - a equipe executora.

Conclusão: 0 aplicativo é uma tecnologia inédita aplicável a essa clientela que pode contribuir ao trabalho do enfermeiro, direcionando a assistência a mulheres em situação de violência sexual por meio do processo de enfermagem.

\section{Abstract}

Objective: To develop an application to support the nursing process in assisting women in situations of sexual violence. Methods: This is an applied research of technological production divided into three stages: elaboration of theoretical material through literature review; assessment of nursing diagnoses and interventions; mobile app development.

Results: The application "CuidarTech ${ }^{\circledR}$ EnfPorElas" presents three navigation options: Nursing diagnoses and interventions, with 33 diagnoses and 613 nursing interventions, correlated and relevant to assist women in situations of sexual violence; Nursing Process, showing theoretical contents about the Nursing Process; Credits, the executing team.

Conclusion: The application is an unprecedented technology applicable to this clientele that can contribute to nurses' work, directing assistance to women in situations of sexual violence through the nursing process.

\section{Resumen}

Objetivo: Desarrollar una aplicación que sirva como apoyo en el proceso de atención de enfermería a mujeres en situación de violencia sexual.

Métodos: Investigación aplicada de producción tecnológica dividida en tres etapas: elaboración del material teórico mediante revisión bibliográfica, evaluación de los diagnósticos e intervenciones de enfermería y desarrollo de la aplicación móvil. 
Resultados: La aplicación “CuidarTech ${ }^{\circledR}$ EnfPorElas" presenta tres opciones de navegación: "Diagnósticos e intervenciones de enfermería” con 33 diagnósticos y 613 intervenciones de enfermería, correlacionados y relevantes para la atención a mujeres en situación de violencia sexual; "Proceso de enfermería" que exhibe contenidos teóricos sobre el proceso de enfermería, y "Créditos" al equipo ejecutor.

Conclusión: La aplicación es una tecnología inédita aplicable a este tipo de clientes que puede contribuir al trabajo del enfermero y orientar la atención a mujeres en situación de violencia sexual mediante el proceso de enfermería.

\section{Introdução}

A violência de gênero constitui um sério problema de saúde pública considerada multicausal e multifatorial, que afeta não só as mulheres, mas também os filhos e familiares, contribuindo para a dinamização da violência. ${ }^{(1)}$ É importante destacar que a violência sexual é uma das formas mais cruéis de violência de gênero, que ocorre através do aviltamento do corpo e dos valores da mulher. Esse tipo de abuso geralmente é acompanhado de outros tipos de agressóes, como a violência física e a psicológica. ${ }^{(1,2)}$

$\mathrm{O}$ peso da violência contra as mulheres praticada pelo parceiro íntimo e da violência sexual fez com que muitos países reconhecessem as consequências e a extensão do problema. Com isto, promulgaram leis para criminalizar a violência sexual e a praticada pelo parceiro íntimo, providenciaram serviços legais, de saúde e sociais às mulheres que sofreram abusos porém registram-se poucos esforços para a prevenção da violência contra a mulher. ${ }^{(3)}$

A violência sexual é considerada como qualquer conduta que imponha a mulher presenciar, manter ou participar de relação sexual não desejada, seja por intimidação, ameaça, coação ou uso da força; que a induza a comercializar ou a utilizar, de qualquer modo, a sua sexualidade, impedindo ou limitando o exercício dos seus direitos sexuais e reprodutivos por meio de coação, chantagem, suborno ou manipulação, conforme descrito na Lei Maria da Penha (Lei 11.340/2006). ${ }^{(4)}$

No Brasil, em 2017, foram registrados em torno de 61 mil estupros, com crescimento de 10,1\% em relação ao ano de 2016. Esses dados são apenas a ponta do iceberg, uma vez que há uma fragilidade na contabilização das incidências e prevalências da violência sexual em decorrência da subnotificaçáo dos casos, pois muitos não chegam aos serviços de saúde e de segurança pública. ${ }^{(5,6)}$

No âmbito do impacto desse agravo, observa-se que mulheres que sofrem violência sexual carregam inúmeras consequências, como danos em seu bem-estar físico, sexual, reprodutivo, emocional, mental e social. Além disso, esse tipo de violência pode acarretar gravidez indesejada, abortamentos e infecçôes sexualmente transmissíveis (IST), danos que podem vir acompanhados de sentimento de culpa, vergonha e medo. ${ }^{(2,3,7)}$

Nesse cenário, mulheres em situação de violência sexual requerem um cuidado que necessita de tempo, escuta e respeito. No serviço de saúde, geralmente, o enfermeiro é o primeiro a ter contato com a vítima e, assim, exerce um papel fundamental no atendimento. Dessa maneira, o enfermeiro deve estar capacitado para promover o acolhimento humanizado e o manejo adequado junto com a equipe multidisciplinar. ${ }^{(8)}$

O Processo de Enfermagem (PE) deve ser utilizado para prestar uma assistência de qualidade, segura e humanizada, capaz de atender a mulher em todos os seus aspectos físicos e emocionais. $\mathrm{O} \mathrm{PE}$ é um instrumento que norteia as decisóes clínicas do enfermeiro, organizando o cuidado com açóes efetivas e individuais. ${ }^{(9,10)}$

A utilizaçáo de softwares, como um tipo de tecnologia, que contemplem as etapas do $\mathrm{PE}$, podem facilitar o trabalho do enfermeiro e o direcionamento da atuaçáo da equipe de enfermagem como apoio à decisão clínica considerando um conjunto de processos concretizados a partir de experiências diárias e de pesquisas que desenvolvem o conhecimento organizado e articulado com uma finalidade prática específica de gerar conhecimento e transformar uma utilização empírica em científica. ${ }^{(11,12)}$

Nesse enquadramento, visando prover um cuidado sistemático e qualificado, bem como facilitar a documentação em saúde, a comunicação entre os profissionais e a tomada de decisões no cuidado, foi elaborada a seguinte questáo norteadora: "Quais os principais diagnósticos e intervençóes de enfermagem que podem compor uma tecnologia que apoie o processo de enfermagem à mulher vítima 
de violência sexual?". Com isso, este estudo teve por objetivo desenvolver um aplicativo para apoio ao processo de enfermagem na assistência à mulher em situação de violência sexual durante o atendimento hospitalar ou ambulatorial.

\section{Métodos}

Trata-se de uma pesquisa aplicada, de produção tecnológica, concebida em três etapas: levantamento dos sinais e sintomas presentes em mulheres vítimas de violência sexual e dos diagnósticos de enfermagem; avaliação dos Diagnósticos de Enfermagem (DE) e Intervenções de Enfermagem (IE); e desenvolvimento do aplicativo móvel.

\section{Primeira etapa}

Foram levantados os sinais e sintomas presentes em mulheres vítimas de violência sexual e dos diagnósticos de enfermagem através da revisão de literatura utilizando manuais do Ministério da Saúde e da Sociedade Brasileira de Ginecologia e Obstetrícia, bem como diretrizes clínicas das associaçóes nacionais e internacionais de ginecologia e enfermagem obstétrica, artigos, teses e dissertaçóes que tratam da temática violência sexual contra a mulher. Em seguida, foram mapeados os principais sinais e sintomas encontrados nas mulheres vítimas de violência sexual que evidenciassem as necessidades humanas básicas afetadas de acordo com o referencial teórico de Wanda de Aguiar Horta. ${ }^{(13)}$ A partir dos sinais e sintomas, foram identificados os DE na taxonomia da NANDA-I, que junto com o referencial teórico das Necessidades Humanas Básicas de Wanda Horta foram adotados, visto serem aplicados na instituição do estudo.

\section{Segunda etapa}

A segunda etapa consistiu na avaliação dos DE e IE para a mulher em situação de violência sexual. A avaliação dos DE foi realizada por meio de um painel Delphi, entre os meses de setembro a novembro de 2019, com um grupo de especialistas constituído por enfermeiros que atuam no atendimento a mulheres em situação de violência com no mínimo 1 (um) ano de experiência, vinculados a instituiçóes de ensino, pesquisa, assistência ou a órgãos de classe da Enfermagem no Estado do Espírito Santo. Os enfermeiros foram selecionados através da amostragem por conveniência do tipo "bola de neve" (snowball sampling), em que os juízes selecionados anteriormente indicam outros especialistas. $\mathrm{O}$ critério de exclusão foi o enfermeiro estar de férias, licenças ou atestado médico. As cartas convite foram enviadas para 17 juízes, sendo que destes 13 aceitaram participar da pesquisa na primeira rodada e apenas cinco participaram da segunda rodada do painel Delphi. Cada especialista recebeu carta convite e o Termo de Consentimento Livre e Esclarecido (TCLE) pelo correio eletrônico. O formulário foi confeccionado em uma plataforma eletrônica online - (Google forms) que permite a sua visualização pelos juízes apenas após o aceite do TCLE com prazo da devolutiva de 15 dias entre as etapas. Para avaliação da representatividade de cada diagnóstico de enfermagem, era permitido aos juízes assinalarem com um " $\mathrm{x}$ " apenas uma das opçóes, em uma escala tipo Likert contendo: "1 - Concordo" e "2 - Não concordo" e, após a resposta, havia espaço disponível para observações e sugestôes. Depois da devolução dos formulários, os dados foram tabulados utilizando um programa Microsoft ${ }^{\oplus}$ Excel $^{\oplus} 2010$ e os comentários e sugestóes foram organizados em um quadro de análise. Foi levado em consideração o índice de concordância entre os juízes quando superior a $80,0 \%$. Os DE com concordância inferior a 80,0\% foram reenviados, posteriormente, para segunda rodada, conforme o método Delphi sugere, com as devidas justificativas baseadas na revisão de literatura, em resposta às observaçóes levantadas pelos juízes para nova avaliação. Os DE que alcançaram $80,0 \%$ de concordância tiveram as intervenções de enfermagem elaboradas e baseadas em revisóes da literatura e no livro Ligaçóes NANDA-NOC-NIC que trazem níveis de evidências em suas correlaçóes. ${ }^{(14)}$ Considerando que essas ligações já haviam sido realizadas por uma equipe de especialistas ou em pesquisas, foram consideradas como já avaliadas.

\section{Terceira etapa}

A terceira etapa consistiu no desenvolvimento do aplicativo móvel. Para isso, foi utilizado o material 
teórico elaborado anteriormente, composto dos diagnósticos e das intervençóes de enfermagem, para compor as telas e para a concepção do mapa conceitual do aplicativo. Em seguida, foram geradas as alternativas de implementação e prototipagem para definição das funcionalidades do aplicativo. A realização dos testes do aplicativo pela equipe de desenvolvimento levou em consideração a usabilidade e a heurística, compostas por: 1) visibilidade do sistema; 2) correspondência entre o sistema e o mundo real; 3) controle e liberdade do usuário; 4) consistência e padronização; 5) reconhecimento em vez de memorização; 6) flexibilidade e eficiência de uso; 7) projeto estético e minimalista; 8) prevenção de erros do sistema; 9) ajudar os usuários a reconhecerem, diagnosticarem e se recuperarem de erros do sistema; 10) ajuda e documentação; 11) melhoria da interação homem/dispositivo, que deve ser agradável e respeitosa com o usuário; 12) interação física e ergonomia permitindo acesso universal; e 13) legibilidade e layout. ${ }^{(15)}$ As funcionalidades, os fluxos de interação e a lógica de funcionamento do aplicativo foram construídos em parceria entre o Laboratório e Observatório de Ontologias Projetuais (Loop/Ufes), o Laboratório CuidarTech $^{\circledast}$ e o Laboratório de Estudos sobre Violência e Saúde (LAVISA ${ }^{\circledast / U F E S) . ~ A p o ́ s ~ a s ~ c o r-~}$ reções, o aplicativo será registrado. $\mathrm{O}$ estudo foi aprovado no Comitê de Ética em Pesquisa sob CAAE no. 57930016.0.0000.5060.

\section{Resultados}

\section{Caracterização da amostra de juízes}

Dos 13 juízes selecionados, 70\% possuíam experiência superior a cinco anos no atendimento a mulheres vítimas violência. Além disso, 15\% possuíam doutorado, 23\% possuíam mestrado e os demais possuíam especialização em enfermagem. Quanto ao trabalho realizado, $13 \%$ dos enfermeiros eram referências técnicas estaduais para atendimento a mulheres em situação de violência, 13\% enfermeiras docentes de uma universidade federal, $40 \%$ na unidade básica de saúde do estado e 34\% trabalham em hospital.

\section{Avaliação dos Diagnósticos e Intervenções de Enfermagem}

Inicialmente, foi realizado o levantamento dos sinais e sintomas mais comumente encontrados em mulheres em situação de violência sexual e, posteriormente, realizado um mapeamento cruzado com os DE da taxonomia NANDA-I, no qual foram evidenciados 33 diagnósticos. Em seguida, esses diagnósticos foram agrupados em necessidades psicossociais/psicoespirituais (72,7\%) e necessidades psicobiológicas $(27,3 \%)$, conforme proposto por Wanda Horta. Na primeira rodada do painel Delphi, os juízes avaliaram 32 diagnósticos com índice de concordância acima de $80,0 \%$ e o diagnóstico "Controle ineficaz da saúde", que obteve índice de $76,9 \%$, foi validado na segunda rodada. Dessa maneira, $100,0 \%$ dos diagnósticos foram considerados adequados e representativos da assistência de enfermagem à mulher em situação de violência sexual. Após a etapa de avaliação dos diagnósticos, foram elaboradas 613 intervençóes de enfermagem.

\section{Aplicativo "CuidarTech ${ }^{\circledR}$ EnfPorElas"}

Dessa maneira, o aplicativo "CuidarTech ${ }^{\oplus}$ EnfPorElas" foi delineado para apoiar o enfermeiro ou o acadêmico durante o atendimento ambulatorial ou hospitalar à mulher em situação de violência sexual e auxiliar no registro do processo/consulta de enfermagem. $\mathrm{O}$ aplicativo encontra-se em fase de finalização e, após o término da testagem e registro estará disponível gratuitamente para ser carregado nas lojas de aplicativos. A fim de possibilitar o acesso rápido do usuário, foi elaborada uma listagem de diagnósticos com as intervençôes de enfermagem que representam a prática assistencial a essa clientela. A Figura 1 ilustra a tela do aplicativo com o menu principal e as três opçôes de navegação: "Diagnósticos e Intervençôes de Enfermagem", "Processo de Enfermagem"; "Créditos" e "Termos de uso".

A opção "Diagnóstico e Intervenções" contém uma listagem com 33 diagnósticos de enfermagem distribuídos por necessidades humanas básicas, sendo $9 \mathrm{DE}$ relacionados às necessidades psicobiológicas e $24 \mathrm{DE}$ relacionados às necessidades psicossociais/espirituais, correlacionados com 613 interven- 


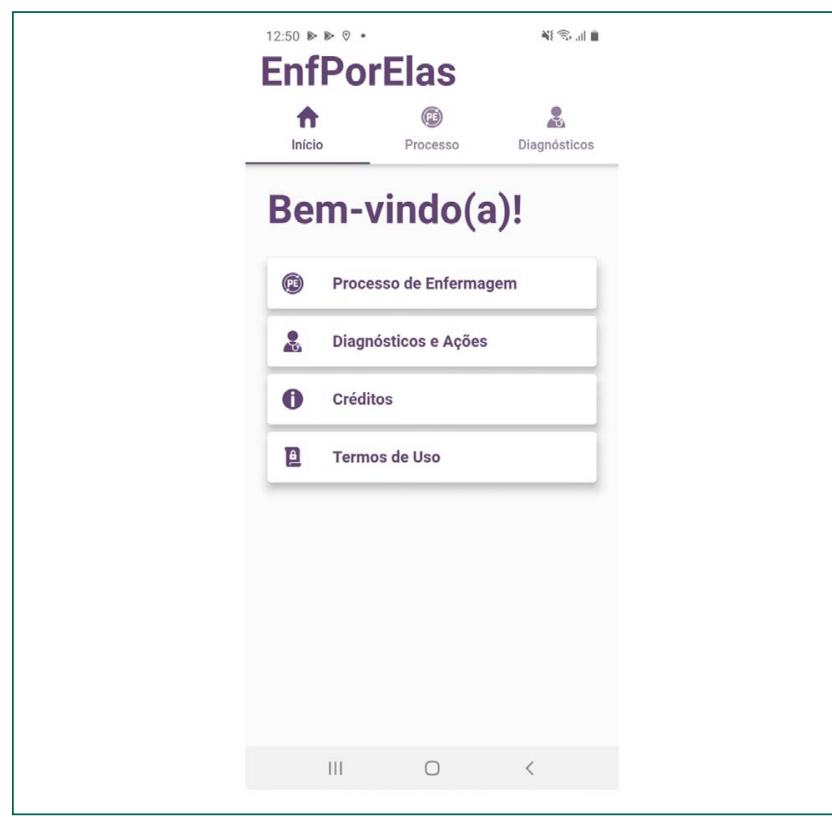

Figura 1. Menu principal do aplicativo "CuidarTech ${ }^{\circledR}$ EnfPorElas"

ções de enfermagem. Ao selecionar um diagnóstico de enfermagem, o usuário visualiza o conceito do diagnóstico, e poderá clicar em "Ver Intervençôes". Ao clicar nesse botáo, surgirá uma listagem com as principais intervençóes de enfermagem para a execuçáo dos cuidados pertinentes ao diagnóstico selecionado, conforme observado no exemplo da Figura 2, com o diagnóstico "Ansiedade", seguindo para as intervençóes.

A opção "Processo de Enfermagem" apresenta conteúdos teóricos com descrição dos conceitos e referências sobre $\mathrm{PE}$ e os tipos de classificação: Processo de enfermagem, Teoria das Necessidades
Humanas Básicas, NANDA-I, NIC, NOC e $\mathrm{CIPE}^{\otimes}$ (Classificação Internacional para a Prática de Enfermagem). Tais conteúdos foram elaborados com base nas últimas publicações acerca dessas temáticas. ${ }^{(14,16-18)}$ No item "Créditos" há informaçôes da equipe responsável pela elaboração do aplicativo e do conteúdo.

\section{Discussão}

O uso de softwares do tipo aplicativo na assistência de enfermagem vem aumentando progressivamente, uma vez que essa ferramenta é capaz de auxiliar o enfermeiro no gerenciamento dos cuidados, favorecendo o conhecimento e familiaridade com as classificações de enfermagem, além de otimizar o tempo de assistência centrada no paciente e maximizar a qualidade do serviço, facilitando o registro e a comunicação entre os membros da equipe. ${ }^{(19)}$

Nesse contexto, o aplicativo "CuidarTech ${ }^{\circledast}$ EnfPorElas" é uma inovação tecnológica assistencial com o propósito de auxiliar o raciocínio clínico e o registro do cuidado à mulher em situação de violência sexual, tanto no atendimento hospitalar quando no ambulatorial, contribuindo para uma assistência humanizada e de qualidade, baseada em evidências científicas, por meio da aplicação do processo de enfermagem.

$\mathrm{O}$ aplicativo proposto utiliza diagnósticos e intervençôes de enfermagem com base em sistema de

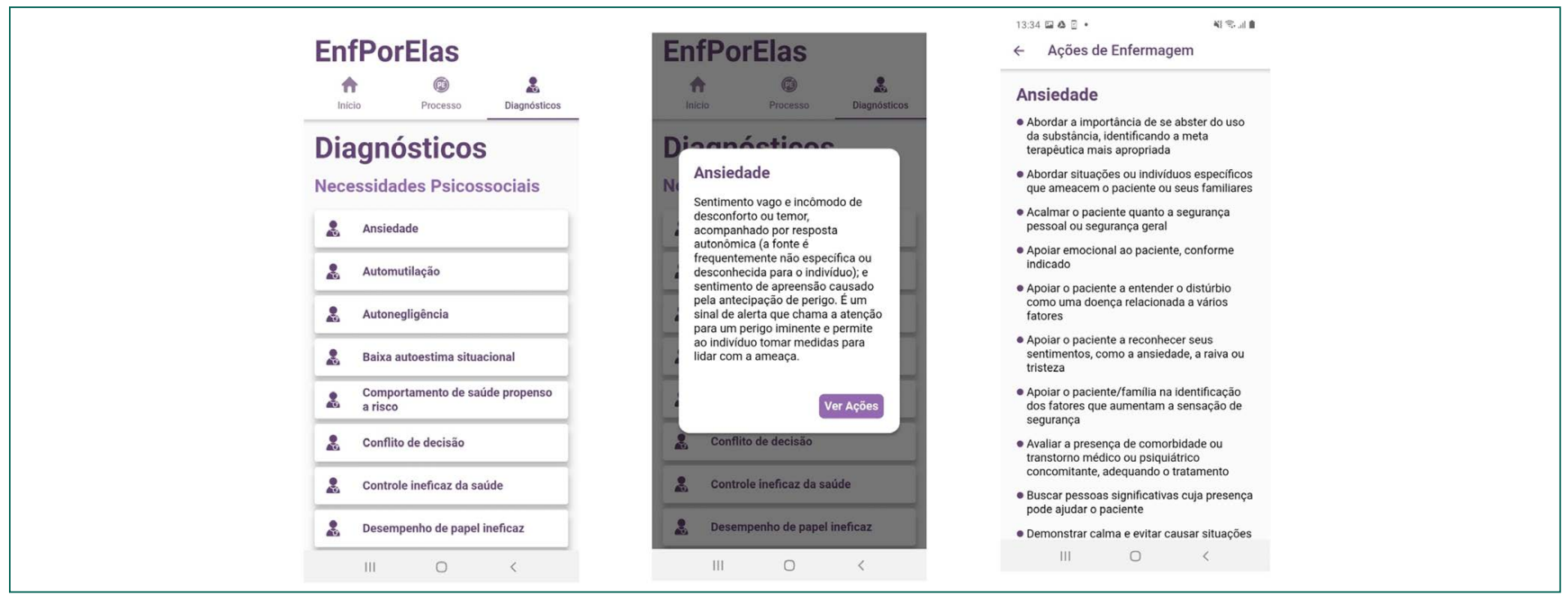

Figura 2. Navegabilidade entre Diagnóstico e Intervenções de Enfermagem 
linguagem padronizada da enfermagem, conforme a ISO 18.104: 2014, que é adequada para apoiar a documentação eletrônica e auxiliar na formaçáo de expressóes diagnósticas e ações de enfermagem. Estudo aponta que diferentes terminologias padronizadas como NANDA-I, CIPEÒ, NIC, seguem a estrutura categorial da Norma ISO 18.104: 2014. A estrutura proposta no aplicativo, os diagnósticos de enfermagem, contém apenas o título com foco, julgamento ou achado clínico. Dessa forma, esses diagnósticos podem ser encontrados tanto na NANDA-I como na CIPEÒ. Em relação às intervenções de enfermagem, as mesmas seguem a estrutura da ISO18.104: 2014. ${ }^{(20)}$

É importante ponderar que os juízes selecionados estavam inseridos em diversos contextos de trabalho, como docência, gerência de saúde, atenção primária e terciária. Alguns dos locais de trabalho, principalmente aqueles do âmbito hospitalar, possuem a aplicação sistemática do processo de enfermagem com a classificação NANDA-I. Entretanto, um profissional da gerência de saúde e dois da atenção primária solicitaram alteraçôes nos enunciados dos títulos dos DE, apesar de saberem dos diagnósticos NANDA-I e da sua importância para a prática de enfermagem, desconheciam como ocorre a sua elaboração e validação. Isto foi evidenciado pelos questionamentos para mudanças na nomenclatura do enunciado dos diagnósticos, o que demonstra o não conhecimento e familiaridade em relação aos diagnósticos propostos pela taxonomia NANDA-I. ${ }^{(21)}$

Todavia, é sabido que os diagnósticos NANDA-I são padronizados e não podem ser modificados de maneira independente pelo enfermeiro, sendo necessário que o mesmo conheça as características definidoras e fatores relacionados para diferenciar um diagnóstico do outro e, assim, as modificações propostas não foram acatadas. ${ }^{(16,17)}$

Vale destacar que ainda há enfermeiros que não se sentem preparados para assistir mulheres em situação de violência. Tal fator implica em insegurança do profissional no atendimento a esse grupo, bem como revela falhas na questão de educaçáo permanente. ${ }^{(8,22)}$

Apesar de compreender as repercussóes da violência sexual, os enfermeiros assumem postura de desconhecimento sobre qual conduta realizar quan- do o cuidado ultrapassa a violência física, sendo esta mais fácil de identificar e de agir conforme modelo tradicional de ensino nas graduaçóes. ${ }^{(23)}$

Propondo uma avaliação clínica com olhar ampliado e integral, o aplicativo "CuidarTech ${ }^{\oplus}$ EnfPorElas” congrega maior número de DE relacionados às necessidades psicossociais/espirituais.

Observa-se, ainda, a dificuldade dos profissionais com a temática, devido à ausência de reflexão e ao desconhecimento do que seja a violência e suas facetas. Isso é resultante da falta de discussáo e abordagem de referenciais teóricos relevantes que forneçam subsídios para a tomada de decisão frente à problemática durante o processo de graduação. Com isso, torna-se imperativa a necessidade de capacitação contínua dos profissionais, promovendo ambientes de discussão e reflexão sobre a violência como problema de saúde pública, a fim de aguçar a sensibilidade para a identificação dos casos e para a arte do cuidar de forma integral e humanizada. ${ }^{24)}$

Nesse sentido, a utilização de um aplicativo pode ajudar enfermeiros e estudantes de enfermagem no desenvolvimento do julgamento clínico e do processo de raciocínio diagnóstico. Além disso, essa tecnologia pode contribuir com os serviços de atendimento às vítimas de violência sexual, ao facilitar a compreensão do enfermeiro sobre a assistência e direcionar a execução e a documentação do processo de enfermagem na sua prática cotidiana. ${ }^{(25)}$

Cabe apontar, que a escolha das intervençóes de enfermagem para cada diagnóstico deve valer-se, primordialmente, do julgamento clínico do enfermeiro. ${ }^{(14)}$

Por fim, a assistência de enfermagem humanizada às mulheres em situação de violência sexual contribui para que as mesmas continuem a viver com dignidade na busca dos restabelecimentos físico e psicológico afetados pela violência. Para isso, é importante que os profissionais visualizem a violência sexual como um problema de saúde pública, realçando o cuidado de enfermagem com açóes de planejamento e mecanismos bem definidos que atendam às necessidades individuais das vítimas. ${ }^{(26)}$

Como limitações do estudo ressalta-se necessidade de realizaçáo de testes de usabilidade com os profissionais da assistência, uma vez que os testes 
foram realizados em ambientes simulados através de um emulador de Android $^{\oplus}$, que é um programa que simula a experiência de executar o sistema operacional de aplicativos feitos para ele, dentro de outro sistema operacional de computador, como, por exemplo, o Windows.

\section{Conclusão}

Este estudo descreveu o desenvolvimento do aplicativo "CuidarTech ${ }^{\circledR}$ EnfPorElas" que é uma ferramenta interativa, objetiva e clara, composta por diagnósticos e intervençóes de enfermagem, que facilita a compreensão do enfermeiro sobre a assistência a vítimas de violência sexual, mitigando o sentimento de impotência do profissional e apoiando seu raciocínio clínico para a execuçáo do processo de enfermagem embasado em uma classificaçáo e teoria de enfermagem, com cuidados fundamentados em evidência científica. $\mathrm{O}$ aplicativo foi considerado confiável e adequado, segundo avaliaçáo de especialistas no assunto e, futuramente, pretende-se desenvolver estudos de aplicação para avaliaçáo dos impactos do uso da tecnologia na assistência de enfermagem. $\mathrm{O}$ aplicativo utiliza diagnósticos e intervençôes de enfermagem conforme a ISO 18.104: 2014, que é adequada para diferentes terminologias padronizadas, como NANDA-I, NIC e CIPEÒ. Considerando a importância do conhecimento técnico para a construção do aplicativo, ressalta-se a contribuição da equipe de design e da ciência da computação na produção interdisciplinar. Essas parcerias têm como potencialidade ampliar os conhecimentos das equipes envolvidas em relação às especificidades de cada área. No entanto, também apresenta o limite de a equipe de saúde precisar compreender conceitos básicos das outras áreas afins para poder desenvolver novas tecnologias na área da saúde.

\section{Agradecimentos}

À Coordenação de Aperfeiçoamento de Pessoal de Nível Superior - Brasil (CAPES) e ao Conselho Federal de Enfermagem - COFEN, através do
Edital CAPES/COFEN no 27/2016 - Código de financiamento 001 .

\section{Colaborações}

Alves OM, Primo CC, Tavares FL, Lima EFA e Leite FMC declaram que contribuíram com a concepção do estudo, desenvolvimento, análise e redação do artigo, bem como participaram da revisão crítica do manuscrito e aprovaram a versão final.

\section{Referências}

1. Cerqueira D, Coelho DS, Ferreira H. Estupro no Brasil: vítimas, autores, fatores situacionais e evolução das notificações no sistema de saúde entre 2011 e 2014. Rev Bras Segurança Pública. 2017;11(1):24-48.

2. Brasil. Ministério da Saúde. Ministério da Justiça. Secretaria de Políticas para as Mulheres. Atenção humanizada às pessoas em situação de violência sexual com registro de informações e coleta de vestígios: norma técnica [Internet]. Brasília (DF): Ministério da Saúde; 2015 [citado 2020 Abr 15]. Disponível em: https://bvsms.saude.gov. br/bvs/publicacoes/atencao_humanizada_pessoas_violencia_sexual_ norma_tecnica.pdf

3. World Health Organization (WHO). Preventing intimate partner and sexual violence against women: taking action and generating evidence [Internet]. Geneva: WHO; 2010 [cited 2020 Feb 02]. Available from: https://apps. who.int/iris/handle/10665/44350

4. Brasil. Presidência da República. Secretaria Geral. Subchefia para Assuntos Jurídicos. Lei $n^{0} 11.340$, de 07 agosto de 2006. Cria mecanismos para coibir a violência doméstica e familiar contra a mulher, nos termos do $\S 8^{\circ}$ do art. 226 da Constituição Federal, da Convenção sobre a Eliminação de Todas as Formas de Discriminação contra as Mulheres e da Convenção Interamericana para Prevenir, Punir e Erradicar a Violência contra a Mulher; dispõe sobre a criação dos Juizados de Violência Doméstica e Familiar contra a Mulher; altera o Código de Processo Penal, o Código Penal e a Lei de Execução Penal; e dá outras providências [Internet]. Brasília (DF): Presidência da República; 2006 [citado 2020 Fev 15]. Disponível em: http://www. planalto.gov.br/ccivil_03/_at02004-2006/2006/lei/111340.htm

5. Brasil. Fórum Brasileiro de Segurança Pública. Activities Report 2018 [Internet]. São Paulo: Fórum Brasileiro de Segurança Pública; 2018 [cited 2020 Feb 15]. Available from: http://www.forumseguranca. org.br/wp-content/uploads/2019/03/Anuario-Brasileiro-deSeguran\%C3\%A7a-P\%C3\%BAblica-2018.pdf

6. Pinto LS, Oliveira IM, Pinto ES, Leite CB, Melo AN, Deus MC. Women's protection public policies: evaluation of health care for victims of sexual violence. Cien Saude Colet. 2017;22(5):1501-8.

7. World Health Organization (WHO). Violence and Injury Prevention. Global status report on violence prevention 2014 [Internet]. Geneva: WHO; 2014 [cited 2020 Apr 16]. Available from: https://www.who.int/ violence_injury_prevention/violence/status_report/2014/en/

8. Baptista RS, Chaves OB, França IS, Sousa FS, Oliveira MG, Leite CC. Sexual violence against women: nurses' practice. Rev Rene. 2015;16(2):210-7. 
9. Cruz DA, Guedes ES, Santos MA, Sousa RM, Turrini RN, Maia MM, et al. Nursing process documentation: rationale and methods of analytical study. Rev Bras Enferm. 2016;69(1):183-9.

10. Martins MC, Batista AC, Amor Divino AE. A Sistematização da Assistência de Enfermagem no atendimento a mulheres vítimas de violência. Cad Graduação: Ciên Biol Saúde. 2018;4(3):113-22.

11. Silva AM, Mascarenhas VH, Araújo SN, Machado RS, Santos AM, Andrade EM. Mobile technologies in the Nursing area. Rev Bras Enferm. 2018;71(5):2570-8.

12. Miranda LN, Farias IP, Almeida TG, Trindade RF, Freitas DA, Vasconcelos EL. Decision-making system for nursing: integrative review. Rev Enferm UFPE Online. 2017;11(Suppl 10):4263-72.

13. Horta VA. Processo de enfermagem. Rio de Janeiro: Guanabara Koogan; 2011.

14. Johnson M, Moorhead S, Bulecheck G, Butcher H, Maas M, Swanson E. Ligações NANDA-NOC-NIC- Condições clínicas. 3a ed. Rio de Janeiro: Elsevier; 2012.

15. Gómez RY, Caballero DC, Sevillano JS. Heuristic evaluation on mobile interfaces: a new checklist. Scientific World J. 2014;2014:1-19.

16. Bulechek GM, Butcher HK, Dochterman JM. Classificação das Intervenções de Enfermagem (NIC). 6a ed. Rio de Janeiro: Elsevier; 2016.

17. Herdman $\mathrm{TH}$, Kamitsuru S. NANDA International Nursing Diagnoses: definitions \& classification, 2018-2020. Porto Alegre: Artmed; 2018.

18. Garcia TR, organizador. Classificação Internacional para a Prática de Enfermagem (CIPE): versão 2019. Porto Alegre: Artmed; 2020.
19. Lima JJ, Vieira LG, Nunes MM. Computerized nursing process: development of a mobile technology for use with neonates. Rev Bras Enferm. 2018;71(Suppl 3):1273-80.

20. Marin HF, Peres HH, Dal Sasso GT. Categorical structure analysis of ISO 18104 standard in nursing documentation. Acta Paul Enferm. 2013;26(3):299-306.

21. Castro RR, Alvino CAL, Chaves ES, Moreira RP, Oliveira RL. Conceptions and challenges in the systematization of nursing care. Rev Enferm UERJ. 2016;24(5):e1046.

22. Perucci M, Gomes MF, Reticena KO, Carvalho VC, Santos MS, Reis FD, et al. Percepções de enfermeiros sobre 0 atendimento à vítimas de violência sexual. Enferm Rev. 2019;22(1):1-11.

23. Bezerra JF, Silva RM, Cavalcanti LF, Nascimento JL, Vieira LJ, Moreira GA. Concepts, causes, and repercussions of sexual violence against women under the optic of healthcare professionals. Rev Bras Promoç Saúde. 2016;29(1):51-9.

24. Silva RM, Arruda CN, Bezerra IC, Castro PC, Vieira LJ, Cavalcanti LF. Processos Formativos de Enfermeiros com Relação à Violência Sexual contra a Mulher. Atas - Investig Qualitativa Saúde. 2018;2:1005-10.

25. Silva KL, Évora YD, Cintra CS. Software development to support decision making in the selection of nursing diagnoses and interventions for children and adolescents. Rev Lat Am Enfermagem. 2015;3(5):92735.

26. Sales ER. Assistência de enfermagem às mulheres vítimas de violência sexual. Rev Científica Multi Nucleo Conhecimento. 2019;1(2):140-58. 